\title{
Ralstonia mannitolilytica infections in an oncologic day ward: description of a cluster among high-risk patients
}

Claudia Lucarelli, ${ }^{1} 2^{*}$ DD, Enea Gino Di Domenico ${ }^{3}$, Luigi Toma ${ }^{3}$, Domenico Bracco ${ }^{3}$, Grazia Prignano ${ }^{3}$, Maria Fortunati ${ }^{3}$, Lorella Pelagalli ${ }^{3}$, Fabrizio Ensoli ${ }^{3}$, Patrizio Pezzotti ${ }^{1}$, Aurora García-Fernández ${ }^{1}$, Annalisa Pantosti ${ }^{1}$ and Loredana Ingrosso $0^{1,2}$

\begin{abstract}
Background: Ralstonia spp, an environmental microorganism, has been occasionally associated with healthcare infections. The aim of this study was to investigate an outbreak caused by Ralstonia mannitolilytica in oncology patients.

Methods: Case definition: Oncology outpatients attending a day ward, with positive blood and/or central venous catheter (CVC) culture for Ralstonia spp from September 2013 - June 2014. We analysed medical records, procedures and environmental samples. R. mannitolilytica was identified by 165 rRNA sequencing, and typed by Pulsed Field Gel Electrophoresis (PFGE); resistance to carbapenemes was investigated by phenotypic and molecular methods.

Results: The patients $(N=22)$ had different malignancies and received different therapy; all had a CVC and 16 patients presented chills and/or fever. R. mannitolilytica was isolated from both blood and CVC $(n=12)$ or only blood $(n=6)$ or CVC tips $(n=4)$. The isolates had indistinguishable PFGE profile, and showed resistance to carbapenems. All the isolates were negative for carbapenemase genes while phenotypic tests suggests the presence of an AmpC $\beta$-lactamase activity,responsible for carbapenem resistance. All patients had had CVC flushed with saline to keep the venous access pervious or before receiving chemotherapy at various times before the onset of symptoms. After the first four cases occurred, the multi-dose saline bottles used for CVC flushing were replaced with single-dose vials; environmental samples were negative for R. mannitolilytica.

Conclusions: Although the source of R. mannitolilytica remains unidentified, CVC flushing with contaminated saline solution seems to be the most likely origin of $R$. mannitolilytica CVC colonization and subsequent infections. In order to prevent similar outbreaks we recommend removal of any CVC that is no longer necessary and the use of single-dose solutions for any parenteral treatment of oncology patients.
\end{abstract}

Keywords: Ralstonia mannitolilytica, Outbreak, Central venous catheter, Oncologic patients, Carbapenem resistance

\footnotetext{
* Correspondence: claudia.lucarelli@iss.it

${ }^{1}$ Istituto Superiore di Sanità Viale Regina Elena, 29900161 Rome, Italy

2European Program for Public Health Microbiology Training (EUPHEM),

European Centre for Disease Prevention and Control, (ECDC), Stockholm,

Sweden

Full list of author information is available at the end of the article
} 


\section{Background}

The genus Ralstonia comprises a group of nonfermentative, Gram-negative bacteria (NFGN) found in moist environments, such as water, soil and plants [1]. Three Ralstonia species, Ralstonia pickettii, Ralstonia insidiosa and Ralstonia mannitolilytica, formerly designated Burkholderia pickettii, Burkholderia solanacearum and Pseudomonas thomasii, respectively, have been recognized as opportunistic human pathogens [1]. Their relevance has been currently re-evaluated because of their ability to survive in different types of disinfectants and to pass through $0.2-\mu \mathrm{m}$ filters that are used to sterilize solutions $[1,2]$.

Ralstonia spp. is reported as a causative agent of bacteremia $[3,4]$, meningitis $[5,6]$, and sepsis $[7,8]$ in immunocompromised patients and of central venous catheter $(\mathrm{CVC})$-associated bacteremia in oncology patients [1-4, 9]. Several hospital outbreaks have been described that were associated with contaminated solutions, including water for injection, saline solutions, disinfectants and antiseptics [1]. Multidrug resistance in NFGN is widely reported in the literature $[10,11]$ and is causing increasing concern because such bacteria may have a role not only as human pathogens but also as potential reservoirs of resistance genes, particularly when they are found in hospital settings. Several studies have described resistance to fluoroquinolones, $3^{\text {rd }}$ generation cephalosporin and carbapenems [1] in isolates belonging to all the three Ralstonia species.

$R$. pickettii is the Ralstonia species most frequently reported in the literature while only a limited number of infections are attributed to $R$. insidiosa and $R$. mannitolilytica [1]. The clinical importance of these two species is probably underestimated because their biochemical patterns are similar to that of $R$. pickettii, making it impossible their distinction based on conventional microbiological tests only $[1,4,12,13]$.

Here we describe an outbreak caused by $R$. mannitolilytica in patients attending a day ward unit in an oncology hospital in Rome occurred from September 2013 June 2014.

\section{Methods}

\section{Hospital setting}

The Istituto Nazionale Tumori Regina Elena - Istituto Dermatologico San Gallicano is a 215-bed hospital located in Rome with approximately 6,700 inpatient admissions and 900,000 outpatients visits per year. The hospital has two oncology day wards (DW-A and DW-B) located in different buildings, each consisting of a single therapy room with 20 bays for chemotherapy infusion and attended by an average of 65 patients/day. Patients are assigned to either ward while the infusion bays are not pre-assigned neither a register for the allocation of patients to bays is in place. One of the units (DW-A) was affected by the outbreak and was the object of the epidemiological investigation. From September 2013 - June 2014, 2485 patients attended DW-A for a total of 20,177 day-hospital visits.

\section{Epidemiological investigation}

We defined a case as a patient attending DW-A from September 2013 - June 2014 that had a blood culture and/or a CVC tip culture positive for Ralstonia spp. with or without symptoms (chills and/or fever). Two of the authors (CL, LI) assisted by an infection control nurse (MF) reviewed all the medical records of the cases to identify any common medical procedure or any occurrence that might have posed a risk for acquiring Ralstonia spp. infection.

\section{Environmental sampling}

Environmental sampling started on 17 October 2013, immediately after Ralstonia spp. had been isolated from the blood culture of the third case. Laboratory technicians collected the samples using commercially available sterile swabs (COPAN Eswabs, Brescia, Italy), following existing departmental guidelines, in the therapy room from furniture and electronic devices $(N=8)$, and in the drug preparation room $(N=8)$ from personal computer, telephone, fax, medicine cabinet, medicine trolley. Swabs were cultured in Tryptic Soy Broth, incubated for $48 \mathrm{~h}$ at $37^{\circ} \mathrm{C}$, and plated on chocolate agar and blood agar. Samples were also obtained from liquid soaps $(N=10)$, their dispensers $(N=8)$ and chlorhexidine $(N=4)$ in use in DW-A. In addition distilled water and sterile water used for injection were cultured as described in Moreira et al. [14].

\section{Microbiological methods and typing of isolates}

Blood samples were taken through CVC in all patients. $R$. mannitolilytica was isolated from signal-positive blood culture bottles (BacT/ALERT BioMérieux, Florence, Italy) using conventional methods. Sixteen CVC tips were cultured according to Zhang et al. [15].

Identification of the isolates was obtained by VITEK 2 system (BioMérieux, Florence, Italy). Identification at the species level was obtained by amplification of the $16 \mathrm{~S}$ rDNA gene followed by double-strand sequencing [16].

To investigate the relatedness of Ralstonia isolates, PFGE was performed on all isolates obtained from blood cultures and CVC tip cultures, following digestion of genomic DNA by the restriction enzyme SpeI (New Englands Biolabs, Ipswich, MA), according to the CDC protocol (available at https://www.cdc.gov/pulsenet/pdf/ecolishigella-salmonella-pfge-protocol-508c.pdf) with addition of $50 \mu \mathrm{M}$ thiourea in the agarose gel and in the electrophoresis running buffer. The following 
running conditions were used: 120 costant angle at $6 \mathrm{~V} /$ $\mathrm{cm}$, with pulse time $20 \mathrm{~h} 1 \mathrm{~s}-40 \mathrm{~s}$, $4 \mathrm{~h} 30 \mathrm{~s}-60 \mathrm{~s}$. Salmonella Braenderup H9812 was used as reference for molecular size.

\section{Antibiotic susceptibility of the isolates}

Susceptibility testing of the isolates was performed by the disk diffusion method according to the EUCAST guidelines (available at http://www.eucast.org/clinical_breakpoints/). The antimicrobials tested were: ceftazidime, meropenem, ciprofloxacin, gentamicin, amikacin, and piperacillin/ tazobactam (Becton Dickinson, Milan, Italy). Antimicrobial susceptibility to piperacillin/tazobactam was confirmed by Etest (BioMérieux, Florence, Italy). As there are no EUCAST susceptibility breakpoints available for Ralstonia spp., the results were interpreted using the EUCAST criteria for Pseudomonas spp. Pseudomonas aeruginosa ATCC 27853 was included as control.

\section{Identification of determinants of carbapenem-resistance}

Four outbreak isolates (A, E, L, S) were randomly selected to perform the phenotypic and molecular tests. Identification of resistance mechanisms for carbapenems was performed by the agar tablet/disc diffusion method (KPC/ MBL and OXA-48 Confirm Kit, ROSCO Diagnostica A/S, Taastrup, Denmark). In addition, PCR assays were performed for the identification of the chromosomal genes bla $a_{\mathrm{OXA}-443}$ and bla $_{\mathrm{OXA}-444}$, previously described in a carbapenem-resistant $R$. mannitolilytica strain [17]. For amplification of the two genes, two couple of primers were designed: OXA-443 Fw 5'-ATGACGAAACTCC GCCA-3'/OXA-443 Rv 5'-AGGTGGGCTCGATCTTG-3' and OXA-444 Fw 5'-ATGTTCTCTCGTTGGTC-3'/ OXA-444 Rv 5'- TGCGGGTCGGACGGAGA -3'. The presence of other carbapenem-resistance genes was investigated by multiplex PCR assay with primers designed to amplify the following 11 genes: $b l a_{\mathrm{IMB}} b l a_{\mathrm{VIM}}, b l a_{\mathrm{NDM}}$, bla $a_{\mathrm{SPM}}, b l a_{\mathrm{AIM}}, b l a_{\mathrm{DIM}}, b l a_{\mathrm{GIM}}, b l a_{\mathrm{SIM}}, b l a_{\mathrm{KPC}}, b l a_{\mathrm{BIC}}$, and $b l a_{\mathrm{OXA}-48}$, accordingly to Poirel et al. [18].

\section{Control strains}

All the experiments were performed using two controls strains: R. mannitolilytica BK931 [2] and R. mannitolilytica ATCC BAA-716 (LMG 6866) [13].

\section{Results}

Epidemiological and microbiological investigation

According to case definition, we identified 22 patients (attack rate 0,88\%), 13 males and 9 females, age range 30-84 years old (median age 66), attending DW-A from 24 September 2013 - 23 June 2014 (Fig. 1, Table 1). In particular, 12 patients had Ralstonia spp. positive cultures from both blood and the CVC tip; four patients didn't have blood culture performed, but because of the clinical symptoms their catheters were removed and cultured and were found positive for Ralstonia spp.; other six patients had a positive blood culture but the CVC tips were not tested.

Analysis of the medical records revealed that the patients had different types of CVC, in particular 20 had a Port, while two had a PICC (percutaneous introduction central catheter). We were able to retrieve the insertion dates of the devices for 13 out of 22 patients: they were inserted from more than 4 years to less than 2 months before the onset of symptoms (Table 1). The patients had different types of solid cancer, they underwent different therapeutic protocols and attended DW-A in different days. The only common procedure among the patients that we were able to identify was a maintenance procedure, which consisted in flushing saline solution through the CVC, after disinfection with $2 \%$ chlorhexidine gluconate in $70 \%$ isopropyl alcohol. Such procedure was performed either before the patient received chemotherapy or as a periodic standard procedure to keep the patient's venous access pervious. Sixteen patients reported fever and/or chills while receiving chemotherapy through $\mathrm{CVC}$ or within two hours after the CVC flushing procedure. No information was available for the remaining six patients. No patient had signs of skin infection at the Port

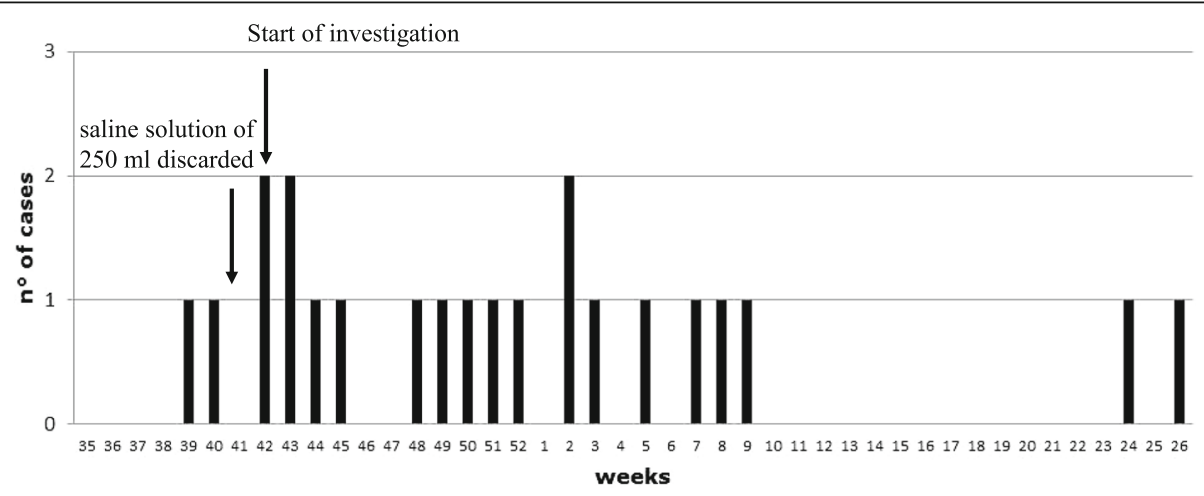

Fig. 1 Epidemic curve of cases of R. mannitolilytica in oncology patients attending the day hospital ward A, September 2013-June 2014 
Table 1 Data of the 22 cases (A-V) of R. mannitolilytica outbreak, September 2013-June 2014, oncology hospital in Rome

\begin{tabular}{|c|c|c|c|c|c|c|c|}
\hline Patient & Sex & Age & Type of CVC & Date of implantation & Date of diagnosis & Blood culture & Tip CVC culture \\
\hline A & $\mathrm{F}$ & 62 & PICC & 08/08/2013 & $24 / 09 / 2013$ & + & ND \\
\hline B & $M$ & 56 & Port & 07/06/2011 & 02/10/2013 & + & ND \\
\hline C & M & 84 & Port & $18 / 09 / 2012$ & $16 / 10 / 2013$ & + & + \\
\hline D & M & 66 & Port & $02 / 04 / 2013$ & $16 / 10 / 2013$ & + & + \\
\hline E & $\mathrm{F}$ & 66 & Port & $13 / 12 / 2011$ & 24/10/2013 & + & + \\
\hline $\mathrm{F}$ & $\mathrm{F}$ & 62 & PICC & $10 / 06 / 2013$ & $24 / 10 / 2013$ & + & ND \\
\hline G & $\mathrm{F}$ & 73 & Port & $31 / 05 / 2011$ & 28/10/2013 & + & + \\
\hline $\mathrm{H}$ & $\mathrm{F}$ & 65 & Port & NA & 07/11/2013 & + & + \\
\hline । & M & 67 & Port & $13 / 12 / 2011$ & 29/11/2013 & + & + \\
\hline J & M & 56 & Port & NA & 03/12/2013 & + & + \\
\hline K & $\mathrm{F}$ & 30 & Port & $25 / 09 / 2012$ & $14 / 12 / 2013$ & + & + \\
\hline L & $\mathrm{F}$ & 65 & Port & NA & 20/12/2013 & ND & + \\
\hline M & $M$ & 62 & Port & 10/10/2010 & $23 / 12 / 2013$ & + & + \\
\hline$N$ & F & 72 & Port & NA & 07/01/2014 & + & + \\
\hline $\mathrm{O}$ & $M$ & 77 & Port & NA & 09/01/2014 & + & ND \\
\hline$P$ & $\mathrm{~F}$ & 67 & Port & NA & $14 / 01 / 2014$ & ND & + \\
\hline Q & $M$ & 80 & Port & $15 / 04 / 2009$ & $30 / 01 / 2014$ & + & ND \\
\hline $\mathrm{R}$ & $\mathrm{F}$ & 80 & Port & NA & $14 / 02 / 2014$ & + & ND \\
\hline S & $\mathrm{F}$ & 63 & Port & NA & $21 / 02 / 2014$ & + & + \\
\hline T & $M$ & 70 & Port & NA & $27 / 02 / 2014$ & + & + \\
\hline$U$ & $M$ & 61 & Port & $31 / 01 / 2013$ & $12 / 06 / 2014$ & ND & + \\
\hline V & $M$ & 61 & Port & $05 / 12 / 2011$ & $23 / 06 / 2014$ & ND & + \\
\hline
\end{tabular}

$N A$ : not available; $N D$ : not determined

site or at the PICC insertion site. Empirical antibiotic therapy (ciprofloxacin) was deemed necessary for one patient only. All patients had their CVCs removed and this led to resolution of symptoms in all cases.

The review of the current medical practices of DWA revealed that, between August 2013 and September 2013, five bottles of saline solution of $250 \mathrm{ml}$ were used for CVC flushing. This type of bottle was in use only in DW-A, and not in DW-B, and usually remained in use for two days from opening. All the cases attended the DW-A for CVC flushing when the $250 \mathrm{ml}$ bottles of saline solution were in use. After the occurrence of the first three cases, the hospital committee for control of healthcare-associated infection gave indications to perform environmental cultures and room disinfection (mid October). However, since the $250 \mathrm{ml}$ bottles of saline solution had been already discarded and replaced with single-dose vials, they were unavailable for microbiological testing when the investigation was started. All the environmental samples and samples from chlorhexidine, distilled water and sterile water were negative for the presence of Ralstonia spp.

\section{Characterization of the pathogen}

Ralstonia spp. had never been isolated from any patients in the hospital both before and after the outbreak. By routine biochemical tests the isolates from blood and CVC tips were identified as $R$. pickettii. However, $16 \mathrm{~S}$ rDNA sequencing showed that the isolates were in fact $R$. mannitolilytica. In addition, all the isolates shared an indistinguishable PFGE profile (Fig. 2) while the two control strains (ATCC BAA-716 and BK931), showed profiles that were distinct from those of the outbreak strains. The outbreak strains had the same multi-drug resistance profile: they were resistant to ceftazidime, meropenem, ciprofloxacin, gentamicin, and amikacin but were susceptible to piperacillin/tazobactam (MIC $\leq 16$ $\mathrm{mg} / \mathrm{L}$ ). Except for susceptibility to ciprofloxacin, the two control strains had the same resistance pattern.

Regarding resistance to carbapenems the strains were negative for the presence of the 11 carbapenemase genes screened by multiplex PCR. All the isolates tested, including the two controls, were positive by PCR for the $b l a_{\mathrm{OxA}}$ 443 and $b l a_{\text {OXA-444 }}$ genes. Phenotypic tests performed to assess the mechanism of carbapenem resistance showed that cloxacillin was able to abolish meropenem resistance, 




Fig. 2 Pulsed-field gel electrophoresis (PFGE) of R. mannitolilytica strains. M: Molecular weight standard, Salmonella Braenderup H9812. A: strain isolated from blood culture. $U, V$ : strains isolated from CVC. These strains are representative of the entire sample. BK931, ATCC BAA-716: control strains

thus suggesting the presence of an AmpC $\beta$-lactamase activity.

\section{Discussion}

The microbiological and epidemiological investigation of this outbreak did not detect the source of the contamination, however the molecular typing of the pathogen strongly supports the hypothesis of a common source of contamination. Failure to identify the culprit is probably due to the fact that when the investigation was started samples from disinfectants, antiseptics and saline solutions used at the beginning of the outbreak were not available for microbiological investigation. In fact, disinfectants, antiseptics and multi-dose bottles of saline solution have been described in the literature as one of the main sources for Ralstonia spp. contamination [1], therefore their prompt removal from DW-A immediately after the beginning of the outbreak was the most understandable precaution to adopt from the patients' safety viewpoint. However, in spite of the multi-dose bottles of saline solution removal, the outbreak lasted 9 months. Indeed, the revision of the medical records showed that CVC flushing with saline solution was a common procedure adopted with all the patients and that, at the beginning of the outbreak, multi-dose bottles of saline solution were in use in DW-A. Thus it is very likely that patients were exposed to one or more contaminated bottle(s) of saline. The majority of the patient developed clinical symptoms immediately thereafter, while others likely had a CVC colonization by $R$. mannitolilytica. Any subsequent procedure through $\mathrm{CVC}$, chemotherapy or new saline flushing, may have caused detachment and dissemination of $R$. mannitolilytica from CVC, causing fever and chills. This might have happened even after several weeks from CVC colonization, possibly accounting for the protracted duration of the outbreak. A similar finding have been reported by Raveh et al. [19] in which, a patient with a CVC developed fever and chills and his blood culture was positive for $R$. pickettii. The patient was treated with antibiotic therapy and the CVC was left in place. However 15 months later the patient developed another bloodstream infection with the same organism likely colonizing $\mathrm{CVC}$.

The ability to grow as biofilm on abiotic surfaces plays an important role in colonization of hospital equipment and indwelling medical devices such as CVCs [20]. Ability to form biofilm has been described in different Ralstonia species including also R. mannitolylitica [21-24]. It is conceivable that biofilm formation might have played a role for these strains to allow adherence to CVCs and subsequent dissemination in the host, following the flushing procedures of the device.

The prolonged duration of this outbreak is not unprecedented for this pathogen. Daxboeck et al. [4] reported isolation of $R$. mannitolilytica in 30 patients attending 15 different wards between February 2002 and March 2004; in this study also the source was not identified. Other outbreaks have been reported in pediatric patients, associated with oxygen delivery devices $[25,26]$ and in oncology patients $[1,2]$. In the outbreak described by Gröbner et al. [2], which lasted 11 weeks, the source was not identified but it was hypothesized 
that a contaminated solution administered through CVC could have been the culprit.

It is important to underline that the misuse of multidose vials, as in our outbreak, has been frequently reported as a cause of numerous outbreaks [27-30] in different European countries as well as in USA where the CDC is aware of at least 49 outbreaks occurred in 13 years [31]. Therefore the correct use of single dose vials, for a single patient, is an effective and important way to prevent outbreaks as reported by specific guidelines [32].

$R$. mannitolilytica is frequently recognized as a multiresistant microorganism [4, 33-35] but limited data are available about carbapenem-resistance. To the best of our knowledge, only a limited number of studies so far, have tested susceptibility to this drug: in four studies, all the strains tested were resistant (five in one study and one strain each in the other three) $[2,17,33]$ while Daxboeck et al. [4] reported carbapenem-resistance in 12 strains out of 30. In our isolates no carbapenemase genes were detected, but they were positive for the chromosomal genes $b l a_{\mathrm{OXA}-443}$ and $b l a_{\mathrm{OXA}-444}$, that have been previously described in one $R$. mannitolilytica carbapenem-resistant strain [17] and that bear close similarity to the bla ${ }_{\mathrm{OXA}-22}$ and $b l a_{\text {OXA-60 }}$ genes found in $R$. pickettii [36]. bla $a_{\text {OXA-22 }}$ and $b l a_{\text {OXA-60 }}$ code for two oxacillinase, a narrow spectrum oxacillinase and an inducible carbapenemase, respectively, although none of them was found to be responsible for carbapenem resistance in $R$. pickettii [36, 37]. In addition, the cloxacillin test suggested that the $R$. mannitolilytica isolates were positive for the presence of AmpC $\beta$-lactamases. These enzymes are clinically important because they may confer resistance to a wide variety of $\beta$-lactam drugs, narrow-, expanded-, and broad-spectrum cephalosporins, $\beta$-lactam- $\beta$-lactamase-inhibitor drugs combinations, as well as to aztreonam and carbapenems in case of association with altered porins and/ or efflux mechanisms [38]. Taken together, our data suggest that meropenem resistance was likely due to overproduction of AmpC $\beta$-lactamase, possibly in synergy with a second mechanism (e.g. decreased production of the porin channel and/or activation of efflux systems). Recently, a serin-hydrolase class $C$ family $\beta$-lactamase has been identified in $R$. mannitolilytica (GenBank accession number: WP_045219476) [15], therefore production of this enzyme may contribute to carbapenem resistance.

Although $R$. mannitolilytica did not cause lifethreatening infections in this outbreak as well as in previously reported outbreaks $[2,26]$, the increased detection of Ralstonia spp. in healthcare settings coupled with the emergence of multi-resistant strains of $R$. mannitolilytica, represent a reason of concern, particularly in case of vulnerable patients which may require an antimicrobial therapy.

\section{Conclusion}

We reported the first outbreak due to $R$. mannitolilytica in oncology patients bearing CVC in Italy. Although the source of the outbreak could not be readily identified, the investigation suggested that contaminated saline solution used for CVC flushing may have been the source of the outbreak. In order to prevent possible infections we recommend the removal of any CVC that is not longer necessary and the use of single dose solutions for any parenteral treatment of cancer patients.

\section{Abbreviations \\ CVC: Central venous catheter; DW-A: Day ward A; DW-B: Day ward B; MIC: Minimal inhibitory concentration; NFGN: Non-fermentative gram- negative bacteria; PCR: Polymerase chain reaction; PFGE: Pulsed field gel electrophoresis; PICC: Percutaneous introduction central catheter}

\section{Acknowledgements}

We are grateful to Dr Aftab Jasir for encouraging discussion and helpful suggestion. We would like to thank Dr. Berit Schulte for providing us strain BK931.

\section{Funding}

This work was partially supported by "L'Associazione Nazionale Contro le Infezioni Ospedaliere" (L'ANCIO).

Availability of data and materials

Please contact author for any requests.

\section{Authors' contributions}

$C L$ performed PFGE, antimicrobial susceptibility test and initial draft preparation. $L T, D B, G P, E G D D, M F, L P, F E C L, A P, L I, P P$, participated in the outbreak investigation. AGF performed carbapenem resistance characterization. LI, AP, PP, EGDD, FECLfinalized the manuscript. All authors read and approved the final manuscript.

\section{Competing interests}

The authors declare that they have no competing interests.

\section{Consent for publication}

Not applicable.

Ethics approval and consent to participate

Not applicable.

\section{Author details}

${ }^{1}$ Istituto Superiore di Sanità Viale Regina Elena, 29900161 Rome, Italy. ${ }^{2}$ European Program for Public Health Microbiology Training (EUPHEM), European Centre for Disease Prevention and Control, (ECDC), Stockholm, Sweden. ${ }^{3}$ Istituto Nazionale Tumori Regina Elena, Istituto Dermatologico San Gallicano, Rome, Italy.

Received: 26 October 2016 Accepted: 21 January 2017

Published online: 07 February 2017

\section{References}

1. Ryan MP, Adley CC. Ralstonia spp.: emerging global opportunistic pathogens. Eur J Clin Microbiol Infect Dis. 2014;33(3):291-304.

2. Gröbner S, Heeg P, Autenrieth IB, Schulte B. Monoclonal outbreak of catheter-related bacteraemia by Ralstonia mannitolilytica on two haematooncology wards. J Infect. 2007;55(6):539-44.

3. Mikulska M, Durando P, Molinari MP, Alberti M, Del Bono V, Dominietto A, et al. Outbreak of Ralstonia pickettii bacteraemia in patients with haematological malignancies and haematopoietic stem cell transplant recipients. J Hosp Infect. 2009;72(2):187-8.

4. Daxboeck F, Stadler M, Assadian O, Marko E, Hirschl AM, Koller W. Characterization of clinically isolated Ralstonia mannitolilytica strains using random amplification of polymorphic DNA (RAPD) typing and antimicrobial 
sensitivity, and comparison of the classification efficacy of phenotypic and genotypic assays. J Med Microbiol. 2005;54(Pt 1):55-61.

5. Ann HM. An unusual case of bacterial meningitis caused by Burkholderia pickettii. Clin Microbiol Newsl. 1998; 102-3.

6. Bonatti H, Stelzmueller I, Laimer I, Obwegeser A. Ralstonia pickettii meningitis in a child with hydrocephalus. Eur J Pediatr Surg. 2009;19(5):341-2.

7. Marroni M, Pasticci MB, Pantosti A, Colozza MA, Stagni G, Tonato M. Outbreak of infusion-related septicemia by Ralstonia pickettii in the Oncology Department. Tumori. 2003;89(5):575-6.

8. Weist K, Stolze H, Sohr D, Wickmann L, Liebeskind AK, Rüden H, et al. P14.03 Ralstonia pickettii septicemia in pediatric oncology patients associated with the use of contaminated heparin-saline-solution. J Hosp Infect. 2006; 64(Supplement 1):S74.

9. Pasticci MB, Baldelli F, Camilli R, Cardinali G, Colozza A, Marroni M, et al. Pulsed field gel electrophoresis and random amplified polymorphic DNA molecular characterization of Ralstonia pickettii isolates from patients with nosocomial central venous catheter related bacteremia. New Microbiol. 2005;28(2):145-9.

10. Enoch DA, Birkett Cl, Ludlam HA. Non-fermentative Gram-negative bacteria. Int J Antimicrob Agents. 2007;29 Suppl 3:S33-41.

11. McGowan JE. Resistance in nonfermenting gram-negative bacteria: multidrug resistance to the maximum. Am J Med. 2006;119(6 Suppl 1):S2936. discussion S62-70.

12. Ryan MP, Pembroke JT, Adley CC. Differentiating the growing nosocomial infectious threats Ralstonia pickettii and Ralstonia insidiosa. Eur J Clin Microbiol Infect Dis. 2011;30(10):1245-7.

13. De Baere T, Steyaert S, Wauters G, Des Vos P, Goris J, Coenye T, et al. Classification of Ralstonia pickettii biovar 3/'thomasii' strains (Pickett 1994) and of new isolates related to nosocomial recurrent meningitis as Ralstonia mannitolytica sp. nov. Int J Syst Evol Microbiol. 2001;51(Pt 2):547-58.

14. Moreira BM, Leobons MB, Pellegrino FL, Santos M, Teixeira LM, de Andrade ME, Sampaio JL, Pessoa-Silva CL. Ralstonia pickettii and Burkholderia cepacia complex bloodstream infections related to infusion of contaminated water for injection. J Hosp Infect. 2005;60(1):51-5.

15. Zhang L, Morrison M, Rickard CM. Draft Genome Sequence of Ralstonia pickettii AU12-08, Isolated from an Intravascular Catheter in Australia. Genome Announc. 2014;2(1):e0027-14.

16. Eilmus $\mathrm{S}$, Heil M. Bacterial associates of arboreal ants and their putative functions in an obligate ant-plant mutualism. Appl Environ Microbiol. 2009; 75(13):4324-32.

17. Suzuki M, Nishio H, Asagoe K, Kida K, Suzuki S, Matsui M, et al. Genome Sequence of a Carbapenem-Resistant Strain of Ralstonia mannitolilytica. Genome Announc. 2015:3(3)e00405-15.

18. Poirel L, Walsh TR, Cuvillier V, Nordmann P. Multiplex PCR for detection of acquired carbapenemase genes. Diagn Microbiol Infect Dis. 2011;70(1):119-23.

19. Raveh D, Simhon A, Gimmon Z, Sacks T, Shapiro M. Infections caused by Pseudomonas pickettii in association with permanent indwelling intravenous devices: four cases and a review. Clin Infect Dis. 1993;17(5):877-80.

20. Donlan RM. Biofilms and device-associated infections. Emerg Infect Dis. 2001; $7(2): 277-81$

21. Hu JY, Fan Y, Lin YH, Zhang HB, Ong SL, Dong N, et al. Microbial diversity and prevalence of virulent pathogens in biofilms developed in a water reclamation system. Res Microbiol. 2003;154(9):623-9.

22. Kang Y, Liu H, Genin S, Schell MA, Denny TP. Ralstonia solanacearum requires type 4 pili to adhere to multiple surfaces and for natural transformation and virulence. Mol Microbiol. 2002;46(2):427-37.

23. Zhang L, Gowardman J, Morrison M, Krause L, Playford EG, Rickard CM. Molecular investigation of bacterial communities on intravascular catheters: no longer just Staphylococcus. Eur J Clin Microbiol Infect Dis. 2014;33(7):1189-98.

24. Di Domenico EG, Toma L, Christian C, Ascenzioni F, Sperduti I, Prignano G, et al. Development of an in vitro Assay, Based on the BioFilm Ring Test ${ }^{\oplus}$, for Rapid Profiling of Biofilm-Growing Bacteria. Front Microbiol. 2016;7:1429. Published online 2016 Sep 21. doi: 10.3389/fmicb.2016.01429.

25. Block C, Ergaz-Shaltiel Z, Valinsky L, Temper V, Hidalgo-Grass C, Minster N, et al. Déjà vu: Ralstonia mannitolilytica infection associated with a humidifying respiratory therapy device, Israel, June to July 2011. Euro Surveill. 2013; 18(18):20471.

26. Jhung MA, Sunenshine RH, Noble-Wang J, Coffin SE, St John K, Lewis FM, et al. A national outbreak of Ralstonia mannitolilytica associated with use of a contaminated oxygen-delivery device among pediatric patients. Pediatrics. 2007;119(6):1061-8.
27. Pan A, Dolcetti L, Barosi C, Catenazzi P, Ceruti T, Ferrari L, Magri S, et al. An outbreak of Serratia marcescens bloodstream infections associated with misuse of drug vials in a surgical ward. Infect Control Hosp Epidemiol. 2006:27(1):79-82.

28. Nakashima AK, Highsmith AK, Martone WJ. Survival of Serratia marcescens in benzalkonium chloride and in multiple-dose medication vials: relationship to epidemic septic arthritis. J Clin Microbiol. 1987;25(6):1019-21.

29. Harbarth S, Sudre P, Dharan S, Cadenas M, Pittet D. Outbreak of Enterobacter cloacae related to understaffing, overcrowding, and poor hygiene practices. Infect Control Hosp Epidemiol. 1999;20(9):598-603.

30. Muller AE, Huisman I, Roos PJ, Rietveld AP, Klein J, Harbers JB, Dorresteijn JJ, et al. Outbreak of severe sepsis due to contaminated propofol: lessons to learn. J Hosp Infect. 2010;76(3):225-30.

31. Preventing infection from the misuse of vials. The Joint Commission Issue 52, June 16, 2014 https://www.jointcommission.org/assets/1/6/SEA_52.pdf Accessed 9 Jan 2017.

32. Protect Patients Against Preventable Harm from Improper Use of SingleDose/Single-Use Vials. https://www.cdc.gov/injectionsafety/cdcpositionsingleusevial.html. Accessed 9 Jan 2017.

33. Dotis J, Printza N, Orfanou A, Papathanasiou E, Papachristou F. Peritonitis due to Ralstonia mannitolilytica in a pediatric peritoneal dialysis patient. New Microbiol. 2012;35(4):503-6.

34. Maroye P, Doermann HP, Rogues AM, Gachie JP, Mégraud F. Investigation of an outbreak of Ralstonia pickettii in a paediatric hospital by RAPD. J Hosp Infect. 2000;44(4):267-72.

35. Vaneechoutte M, De Baere T, Wauters G, Steyaert S, Claeys G, Vogelaers D, et al. One case each of recurrent meningitis and hemoperitoneum infection with Ralstonia mannitolilytica. J Clin Microbiol. 2001;39(12):4588-90.

36. Nordmann P, Poirel L, Kubina M, Casetta A, Naas T. Biochemical-genetic characterization and distribution of OXA-22, a chromosomal and inducible class D beta-lactamase from Ralstonia (Pseudomonas) pickettii. Antimicrob Agents Chemother. 2000;44(8):2201-4.

37. Girlich D, Naas T, Nordmann P. OXA-60, a chromosomal, inducible, and imipenem-hydrolyzing class $D$ beta-lactamase from Ralstonia pickettii. Antimicrob Agents Chemother. 2004;48(11):4217-25.

38. Wozniak A, Villagra NA, Undabarrena A, Gallardo N, Keller N, Moraga M, et al. Porin alterations present in non-carbapenemase-producing Enterobacteriaceae with high and intermediate levels of carbapenem resistance in Chile. J Med Microbiol. 2012;61(Pt 9):1270-9.

\section{Submit your next manuscript to BioMed Central and we will help you at every step:}

- We accept pre-submission inquiries

- Our selector tool helps you to find the most relevant journal

- We provide round the clock customer support

- Convenient online submission

- Thorough peer review

- Inclusion in PubMed and all major indexing services

- Maximum visibility for your research

Submit your manuscript at www.biomedcentral.com/submit
) Biomed Central 